\title{
Infective endocarditis caused by Brucella melitensis in an HIV-positive patient
}

\author{
Hulliurudurga Srinivasa Setty Natraj Setty, Anindya Sundar Trivedi, Jayashree Kharge, Sathwik Raj, \\ Phani Teja Mundru, Santhosh Jadav, Yeriswamy Mogalahally Channabasappa, Sandeep Shankar, \\ Tagatagere Ramegowda Raghu, Rahul Patil, Cholenahally Nanjappa Manjunath
}

Sri Jayadeva Institute of Cardiovascular Sciences and Research, Bangalore, Karnataka, India

\begin{abstract}
Introduction: Cardiac complications are becoming more critical in patients with human immunodeficiency virus (HIV) infection. The risk of infectious complications in HIV-positive patients has decreased with the availability of highly active antiretroviral therapy, but remains high in developing countries, such as India. HIV patients are at increased risk for recurrent bacterial infections due to acquired immune suppression.

Case presentation: We describe a case of a 45 -year-old HIV-infected male, on antiretroviral therapy for 4 years, with invasive endocarditis. On admission, his CD4+ count was 274 cells/ $\mu l$. The patient was hemodynamically stable on arrival and was in congestive heart failure. Pallor was present with no peripheral signs of infective endocarditis. 2D echocardiogram revealed vegetations on the tips of anterior and posterior leaflets of the mitral valve, severe mitral regurgitation, and moderate tricuspid regurgitation. Blood culture was positive for Brucella melitensis. The patient recovered without any sequel after six weeks of antibiotic therapy (gentamycin intravenously + rifampicin p.o.). The patient remains under regular follow-up.

Conclusions: Brucellosis in general is a difficult diagnosis to make. Therefore, along with diagnosis, treatment is also delayed leading to devastating outcomes. Cardiac involvement occurs in only $2 \%$ of cases but accounts for $80 \%$ of mortality due to brucellosis. Brucella endocarditis should be suspected in HIV patients with endocarditis, who have negative blood cultures and risk of exposure. The most accepted treatment for $B$. endocarditis is a combination of anti-microbial therapy with surgery.
\end{abstract}

HIV AIDS Rev 2021; 20, 2: 144-146

DOI: https://doi.org/10.5114/hivar.2021.107241

Key words: HIV infection, infective endocarditis, Brucella melitensis, CD4+ count, 2D echocardiogram.

\section{Introduction}

There have been several studies, mostly from western countries, on the association between human immunodeficiency virus (HIV)-positive status and infective endocarditis (IE), for which intravenous drug abuse was the main risk factor. Even with advances in diagnosis, IE remains a common cause of hospitalization, with high

Address for correspondence: Dr. H. S. Natraj Setty, MD, DM, FICC, FACC, FRCP, Associate Professor of Cardiology,

Sri Jayadeva Institute of Cardiovascular Sciences and Research,

\#493, 4th Cross, 7th Main, J.P. Nagar 3rd Phase, Bangalore - 69, Karnataka, India, phone: + 91 080-26580051,

fax: + 91 080-22977261, e-mail: drnatrajsetty75@gmail.com

morbidity and mortality rates [1]. IE was initially recognized to be a complication of intravenous drug use in the 1950s [2]. It is a potentially fatal consequence of injecting illicit drugs, such as heroin, cocaine, and methamphetamine. Very few reports exist regarding IE caused by Brucella melitensis in HIV patients without a history of any injectable drug abuse.

Article history:

Received: 15.03.2020

Received in revised form: 16.11 .2020

Accepted: 11.12.2020

Available online: 30.06 .2021
International Journal of HIV-Related Problem

HIV \& AIDS

R e v i e w 


\section{Case presentation}

A 45-year-old male was referred to our institute with a suspicion of refractory heart failure from a primary care hospital. The patient is HIV-1-positive for the last 6 years and on antiretroviral therapy (ART) for the last four years (CD4+ count was 126 cells/ $\mu$ l at that time); on admission, his CD4+ count was 274 cells/ $\mu$ l (viral load 938 copies/ml). Main complaints at presentation to the local hospital included severe fatigue, intermittent low-grade fever, and increasing shortness of breath for the past two months. He was empirically started on intravenous ceftriaxone and supportive care. Pallor was present, with no peripheral symptoms of IE. Blood cultures were taken. His baseline hemoglobin was $7 \mathrm{gm} / \mathrm{dl}$ and creatinine $1.8 \mathrm{mg} / \mathrm{dl}$. ECG showed sinus tachycardia with no other significant abnormality. Chest X-ray PA was within normal limits, and sputum culture was negative for acid-fast bacilli. 2D echocardiogram revealed vegetations on the tips of the anterior and posterior leaflets of the mitral valve (Figure 1). Color Doppler interrogation showed severe mitral regurgitation and moderate tricuspid regurgitation (Figure 2). Blood culture revealed the growth of Brucella melitensis. The strain was sensitive to gentamycin, rifampicin, cotrimoxazole, and resistant to doxycycline. The aortic valve was unaffected with normal biventricular functions. Intravenous gentamycin $60 \mathrm{mg}$ every 8 hours, and rifampicin $600 \mathrm{mg}$ orally, along with diuretics were started; the patient was also started with two units of packed red cell transfusions. The patient improved with reduced fatigue, shortness of breath by $3^{\text {rd }}$ day of hospitalization. TEE was planned, but the patient did not consent to it. After six weeks of antibiotic therapy, the patient was discharged in a hemodynamically stable condition. Repeat 2D-ECHO at the time of discharge showed complete resolution of the vegetation. The patient remains under regular medical follow-up.

\section{Discussion}

Although bacteremia is common in HIV-positive patients, IE is not routinely considered $[3,4]$. IE and HIV are mainly suspected in intravenous drug users; however, it is quite rare in non-IVDA HIV-positive patients $(2 \%)[5,6]$. HIV alone does not appear to increase the susceptibility for IE in sero-positive patients [7]. As there is no significant difference in the clinical presentation of IE between HIVinfected and HIV-negative patients, it is very important to consider a diagnosis of IE even in HIV patients without any history of IVDA [8].

Our patient is HIV-positive; although, the cause of his HIV infection could not be traced, he remained compliant with ART for the last four years., and his recent CD4+ count is 274 cells $/ \mu$ l (viral load 938 copies $/ \mathrm{ml}$ ). Right-sided infective endocarditis is more common in intravenous drug users. In a European series, Staphylococcus aureus was the most common causative IE organism in HIV-infected patients, whereas Brucella melitensis was mainly associated with en-

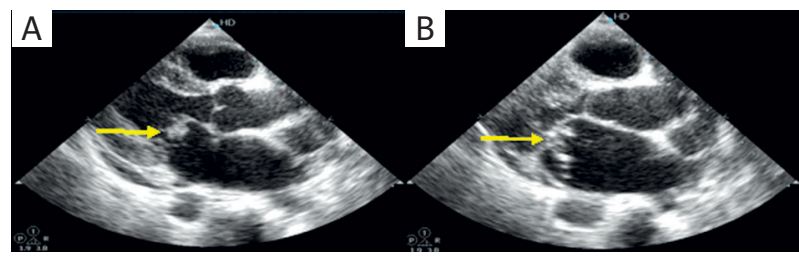

Figure 1. 2D echocardiography with vegetations on the tips of anterior and posterior leaflets of the mitral valve (yellow arrow)

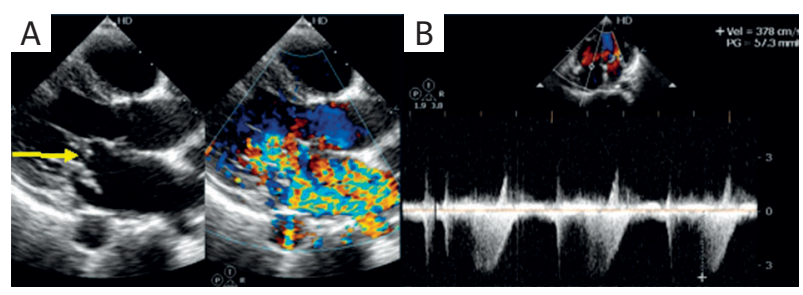

Figure 2. 2D echocardiogram with vegetations on the tips of anterior and posterior leaflets of the mitral valve (yellow arrow), severe mitral regurgitation, and moderate tricuspid regurgitation

docarditis in IVDA patients [9]. Nel et al. [8] also showed no significant differences in the vegetation characteristics between HIV-infected and uninfected patients. They also showed that complications, such as leaflet aneurysms and root abscess occurred in patients with CD4+ counts $<250 /$ cc in HIV-positive subjects, and appeared to be more extensive compared to uninfected patients. The outcomes of pharmacological and surgical therapies were also similar in both groups [8]. The most common presentation of brucellosis is pyrexia of unknown origin, with night sweats, arthralgia, malaise, and asthenia. Cardiovascular involvement most commonly manifests as infective endocarditis but is seen in only $2 \%$ of brucellosis cases [9]. In general, risk factors for B. melitensis infection include intravenous drug abusers, HIV patients, hepatitis $\mathrm{B}$ - and hepatitis C-infected patients, and also people living in rural areas and having exposures to farms' animals [10].

\section{Conclusions}

Infective endocarditis in HIV-positive patients has a similar clinical presentation to those who are HIV-negative, with similar morbidity and mortality. Brucella endocarditis should be suspected in HIV-positive patients with endocarditis, who have negative blood cultures and a risk of exposure. The most accepted treatment for B. endocarditis is a combination of anti-microbial therapy with surgery.

\section{Conflict of interest}

The authors declare no conflict of interest with respect to the research, authorship, and/or publication of this article. 


\section{References}

1. Sousa C, Botelho C, Rodrigues D, et al. Infective endocarditis in intravenous drug abusers: an update. Eur J Clin Microbiol Infect Dis 2012; 9: 2905-2910.

2. Brown P, Levine D. Infective endocarditis in the injection drug user. Infect Dis Clin North Am 2002; 16: 645-665.

3. Netzer RO, Zollinger E, Seiler C, et al. Infective endocarditis: clinical spectrum, presentation, and outcome. An analysis of 212 cases, 1980-1995. Heart 2000; 84: 25-30.

4. Hogevik H, Olaison L, Andersson R, et al. Epidemiologic aspects of infective endocarditis in an urban population: a 5-year prospective study. Medicine 1995; 74: 324-339.

5. Currie PF, Sutherland GR, Jacob AJ, et al. A review of endocarditis in acquired immunodeficiency syndrome and human immunodeficiency virus infection. Eur Heart J 1995; 16 (Suppl B): 15-18.

6. Fernández Guerrero ML, Torres Perea R, Gomez Rodrigo J, et al. Infectious endocarditis due to non-Typhi Salmonella in patients infected with human immunodeficiency virus: report of two cases and review. Clin Infect Dis 1996; 22: 853-855.

7. Nahass RG, Weinstein MP, Bartels J, et al. Infective endocarditis in intravenous drug users: a comparison of human immunodeficiency virus type 1-negative and -positive patients. J Infect Dis 1990; 162 967-970.

8. Nel SH, Naidoo DP. An echocardiographic study of infective endocarditis, with special reference to patients with HIV. Cardiovasc J Afr 2014; 25: 50-57.

9. Kulkarni SK, Bhairappa S, Rangan K. A tale of four valves: outcome of Brucella endocarditis: a case series. Eur Heart J Case Rep 2019; 3: ytz035.

10. Cafardi JM, Haas D, Lamarre T, Feinberg J. Brucella endocarditis in persons who inject drugs. Open Forum Infect Dis 2020; 7: ofaa063. 